\title{
ac aging and space-charge characteristics in low-density polyethylene polymeric insulation
}

\author{
G. Chen ${ }^{\mathrm{a})}$ \\ School of Electronics and Computer Sciences, University of Southampton, Southampton SO17 1BJ, \\ United Kingdom \\ M. Fu \\ Department of Engineering, University of Leicester, Leicester LE1 7RH, United Kingdom

\begin{abstract}
X. Z. Liu and L. S. Zhong
State Key Laboratory for Electrical Equipment and Insulation, Xian Jiaotong University, Xian 711049, China
\end{abstract}

(Received 30 September 2004; accepted 20 January 2005; published online 11 April 2005)

\begin{abstract}
In the present work efforts have been made to investigate the influence of ac aging on space-charge dynamics in low-density polyethylene (LDPE). LDPE films with $200 \mu \mathrm{m}$ were aged under various electric stress levels at $50 \mathrm{~Hz}$ for various times at ambient temperature. Space-charge dynamics in the samples after aging were monitored using the pulsed electroacoustic technique. It has been revealed that the space charge under ac aging conditions is related to the level of the applied field, duration of the voltage application, as well as the electrode materials. By comparing with the results of unaged sample the results from aged sample provide a direct evidence of changing trapping characteristics after ac aging. Negative space charge is present in the bulk of the material and the total amount of charge increases with the aging time. The amount of charge increases with the applied field. Charge decay test indicates that the charges are captured in deep traps. These deep traps are believed to form during the aging and related to change caused by injected charge. By using different electrode materials such as gold, brass alloy, and polyethylene loaded with carbon black, it was found that the electrode has an important role in the formation of charge, hence subsequent changes caused by charge. The charge dynamics of the aged samples under dc bias differ from the sample without ac aging, indicating changes brought in by ac aging. Chemical analysis by Fourier transform infrared spectroscope and Raman microscope reveals no detectable chemical changes taken place in the bulk of the material after ac aging. Finally, the consequence of the accumulation of space charge under ac conditions on the lifetime of the material has been discussed. The presence of deeply trapped space charge leads to an electric stress enhancement which may shorten the lifetime of the insulation system. () 2005 American Institute of Physics. [DOI: $10.1063 / 1.1868880]$
\end{abstract}

\section{INTRODUCTION}

Solid extruded polymeric materials such as crosslinked polyethylene (XLPE) and uncrosslinked polyolefins are used widely for underground high-voltage power transmission cables. The advantages of such materials are their excellent electrical properties combined with good physical properties. While the topic of ultimate failure in polymeric insulation has attracted many interests, ${ }^{1-9}$ many aspects of events prior to the onset of microscopic damage have received less attention. Under certain high-voltage operating conditions, trapped or low mobility electrically charged species within the bulk can give rise to space charge, resulting in localized electric stress enhancement. It has been recognized that the accumulation of space charge in insulating material can cause the deterioration of electrical performance, ${ }^{10}$ may be one of the causes for unexpected breakdown, ${ }^{11}$ and the initiation of electrical trees. ${ }^{12}$

\footnotetext{
a) Author to whom correspondence should be addressed; FAX: +44(0)2380593709; electronic mail: gc@ecs.soton.ac.uk
}

In the past two decades, due to development in mapping the space charge in solid insulation materials, numerous studies have been carried out to pursue a better understanding of the buildup of trapped space charge within solid dielectric materials. ${ }^{13-21}$ Consequently, our knowledge of space-charge dynamics and the factors that can affect charge formation has enabled us to improve the electrical performance of materials via material selection and processing. However, majority of the work has been carried out under dc conditions; little attention has been given to the effect of ac aging on space-charge formation in the bulk insulation. Work on the dynamics and the role of space charge on electrical breakdown under $50-\mathrm{Hz}$ ac conditions has only received limited attention. ${ }^{22-30}$ As the majority of XLPE high-voltage (HV) cables will be operating under ac conditions in the immediate future, it is appropriate to investigate charge trapping and mobility under such conditions. Earlier results using the pulsed electroacoustic technique ${ }^{26}$ revealed that although there was charge accumulation at very low frequencies $(<0.01 \mathrm{~Hz})$ the charge formation at high frequency was negligible especially at power frequency. Al- 
though the measurements were carried out under specific conditions (low-field stress and short period of time), these results gave the impression that space-charge effect under ac conditions is not an issue to be concerned with despite new evidences emerging slowly indicate the effect of space charge on electrical deterioration of the insulating polymers. It is believed that under ac conditions the changes in polarity every half cycle limits the time charge can accumulate when ac frequency is greater than $0.01 \mathrm{~Hz}$. However, if the applied field is high enough and the duration of the voltage application is long enough a small fraction of injected charge can be trapped. Indeed, work using other measurement techniques $^{22-24,27,28}$ demonstrated the presence of space charge in polymeric cable insulation materials under ac conditions. Due to the recombination of majority of electrons and holes alternatively injected each half cycle at $50 \mathrm{~Hz},{ }^{29}$ little quantitative charge distributions were presented. The study of space charge at $50 \mathrm{~Hz}$ at high temperatures using thermal step method ${ }^{30}$ revealed that after 14 days aging at $40-\mathrm{kV} / \mathrm{mm} \mathrm{rms}$ a significant amount of charge has been accumulated in the samples. The amount of charge depends on both materials and temperature. Based on our investigation $^{31,32}$ under dc stressing conditions, the electrode materials play an important role in the formation of space charge in polymeric insulation; different electrode configurations could be another factor contributing to the inconsistency in literatures for ac space charge.

This paper reports on space-charge measurements on 200- $\mu$ m-thick low-density polyethylene (LDPE) using the pulsed electroacoustic (PEA) system. Samples were subjected to $50-\mathrm{Hz}$ ac electric stresses up to $50-\mathrm{kV} / \mathrm{mm} \mathrm{rms}$ at room temperature for a period of more than $50 \mathrm{~h}$ on some occasions. The accumulation of space charge at various times after ac aging was monitored. In addition, the effect of electrode materials under ac condition has also been studied. Aged samples were analyzed using Raman microscopic technique which revealed little chemical change taken place in the material. The charge behavior after ac aging was further studied by applying a dc field. The results clearly demonstrate that the charge dynamics change remarkably after aging. Finally, the possible influence of the accumulated charge on the long-term performance of the material has been discussed.

\section{EXPERIMENTAL DETAILS}

\section{A. Sample details}

LDPE was chosen initially because of its relatively simple chemical structure and its wide applications in cable insulation. Space-charge formation in a material is greatly affected by the presence of impurities and additives as they can act either as ionizable centers under a high electric stress or as trapping sites. In order to reduce the influence of the impurities, additive-free LDPE was selected for the present study. The thickness of the sample was typically $\sim 200 \mu \mathrm{m}$ thick. The diameter of the sample is $50 \mathrm{~mm}$. Sufficient samples were prepared to eliminate the variation from batch

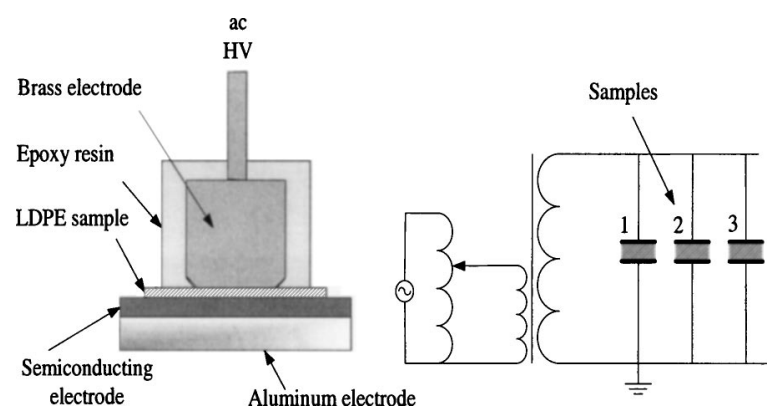

FIG. 1. Schematic diagram of the sample and ac aging test.

to batch. In the case of gold electrodes, Au electrodes with a diameter of $10 \mathrm{~mm}$ were coated on both sides of the sample using the Edwards sputter coating unit.

\section{B. ac electrical aging}

ac electrical aging was carried out at ambient temperature for various times at different applied electric stresses while a majority of the samples were stressed at $50-\mathrm{kV} / \mathrm{mm}$ rms. The values for ac electric stress in this paper are rootmean-square (rms) values unless other stated. In order to prevent from flashover the top brass electrode was cast in epoxy resin. The brass electrode was manufactured from alloy rod (Cu58/Zn39/Pb3) purchased from GoodFellow. The bottom electrode was semicon, made of polyethylene loaded with carbon black (approximately $40 \%$ by weight) to increase its conductivity (approximately $0.5 \mathrm{~S} / \mathrm{m}$ at room temperature). This type of semicon material is commonly used in high-voltage polymeric power cable to eliminate electric stress enhancement and void formation between insulation and metallic electrode. A typical sample and ac aging testing circuit are shown in Fig. 1.

\section{PEA technique}

The space-charge measurements were taken using the PEA system, which has a pulse width of 5 ns. The sensor used was a $9-\mu$ m-thick $\mathrm{LiNbO}_{3}$. A constant pressure was maintained during the measurements. The principle of the PEA technique for plaque and cable geometry is well documented in literature. ${ }^{3-35}$ To summarize, acoustic waves are produced at charge layers at both electrodes and internal charge when an electrical pulse is applied to a sample. The acoustic signals are then detected by a piezoelectric sensor. Through a deconvolution technique and calibration, a quantitative description of charge across the sample can be obtained. The spatial resolution of the system is determined by the pulse width and sensor thickness. A signal processing algorithm has been employed to recover the signal. ${ }^{36}$ In our PEA system for plaque geometry the resolution is less than $10 \mu \mathrm{m}$ which is considered adequate in the present study.

\section{Chemical analysis}

It is well known that long-term aging can cause chemical changes which subsequently deteriorate the electrical performance of the material. High electric stress can accelerate the processes of deterioration. Infrared (IR) and Raman spectra 


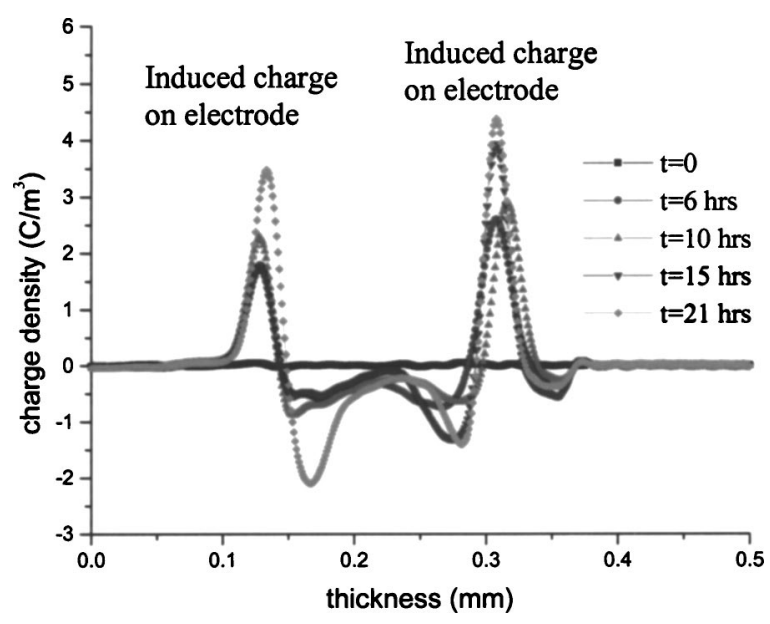

FIG. 2. Charge distribution in ac aged samples vs aging time.

are very sensitive to the chemical changes. They have been used extensively to examine chemical changes caused by electrical aging. In the present study, these techniques have been used to monitor any chemical changes taken place in the aged sample.

\section{EXPERIMENTAL RESULTS}

\section{A. Charge formation during ac aging}

Once the samples were removed from the ac aging at various aging times, the samples were transferred to the PEA system and the space chage formed during the aging was examined without the applied field unless otherwise stated. The transfer time was typically less than $5 \mathrm{~min}$. The charge distributions in the samples aged at $50 \mathrm{kV} / \mathrm{mm}$ for various aging times are shown in Fig. 2. Although charge was measured in the sample aged as long as $56 \mathrm{~h}$, only selected charge distribution profiles were illustrated for clarity.

It is noticed that negative charge was formed in all the samples aged. Although the negative charge tends to spread across the sample, more charge can be found in the region adjacent to the electrodes.

The total absolute amount of charge accumulated in the samples can be calculated based on the charge-density distribution given in Fig. 2.

$$
Q(t)=\int_{0}^{d}|\rho(x, t)| S d x,
$$

where $\rho(x, t)$ is the charge density, $S$ the electrode area, and $d$ the thickness of the sample.

The relationship between the total amount of charge accumulated and the aging time at $50 \mathrm{kV} / \mathrm{mm}$ is shown in Fig. 3.

From this figure it is clear that the total amount of charge in the sample shows an initial rapid increase and then reaches to saturation at a longer aging time. The long-term influence of charge presence on the electrical performance of the material is often related to how deep the charges are trapped. This can be estimated by measuring the change in charge profile after a period of time. Figure 4 shows the charge distributions immediately after aging and $24 \mathrm{~h}$ later in the

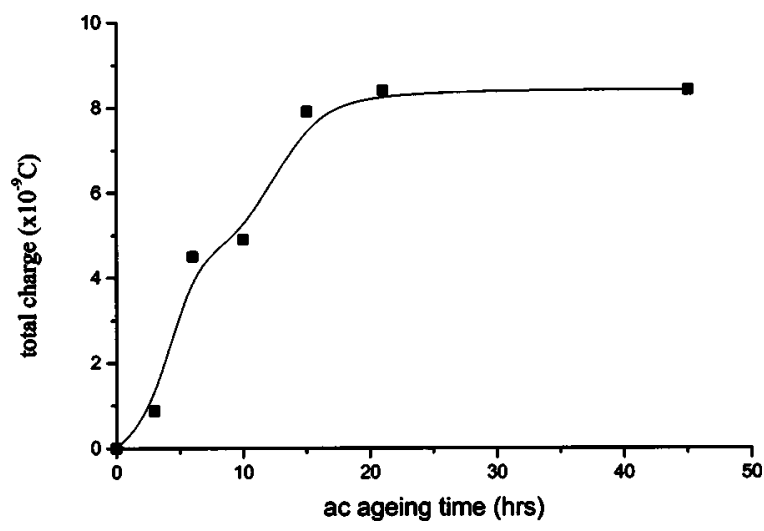

FIG. 3. Total charge in ac aged samples vs aging time.

sample aged for $15 \mathrm{~h}$ at $50 \mathrm{kV} / \mathrm{mm}$. It can be seen that there is no significant change in the distribution profile although the amount of charge is slightly less after $24 \mathrm{~h}$. This is to say that the charge decay rate is very slow, suggesting deeply trapped charges in the bulk.

In order to study the influence of the applied electric stress, samples were aged at different stress levels ranging from 5 to $60 \mathrm{kV} / \mathrm{mm}$ while the aging time was fixed at $45 \mathrm{~h}$. The charge distribution immediately after the removal from aging was measured in the same way, as described earlier, and the total charge remained in the sample was calculated for each sample. Figure 5 shows the total amount of charge in the sample versus the applied ac aging electric stress. As expected, the amount of charge stored in the sample increases with the applied stress. The increase rate accelerates with the applied stress. The result from the sample aged at 60 $\mathrm{kV} / \mathrm{mm}$ was not obtained because all the samples failed prior to reaching $45 \mathrm{~h}$.

\section{B. Electrode effect}

It has been demonstrated ${ }^{31}$ that the electrode and polymeric interface play an important role in the formation of space charge. More importantly, by comparing the effect of the different electrode interfaces on charge distribution and the amount of charge in the material it is possible to identify the source of charge. Samples with gold electrodes on both

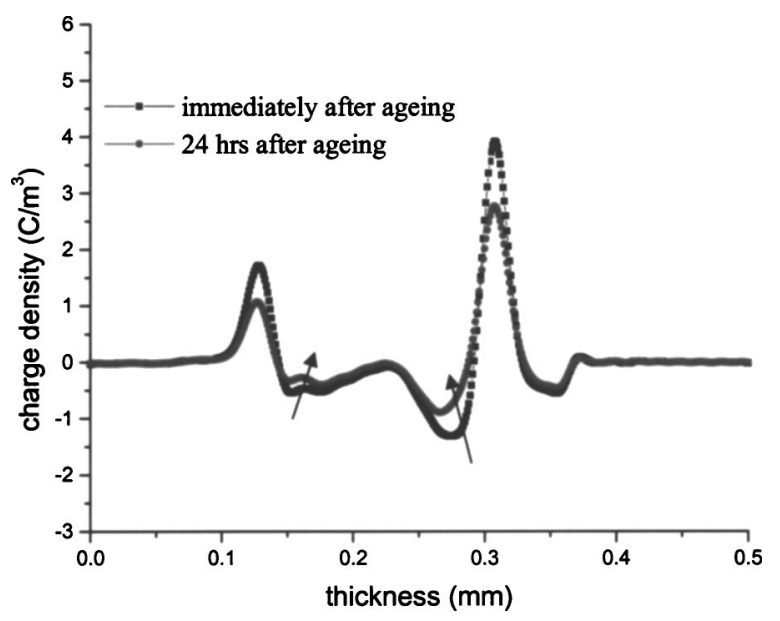

FIG. 4. Evolution of charge distribution after ac aging. 


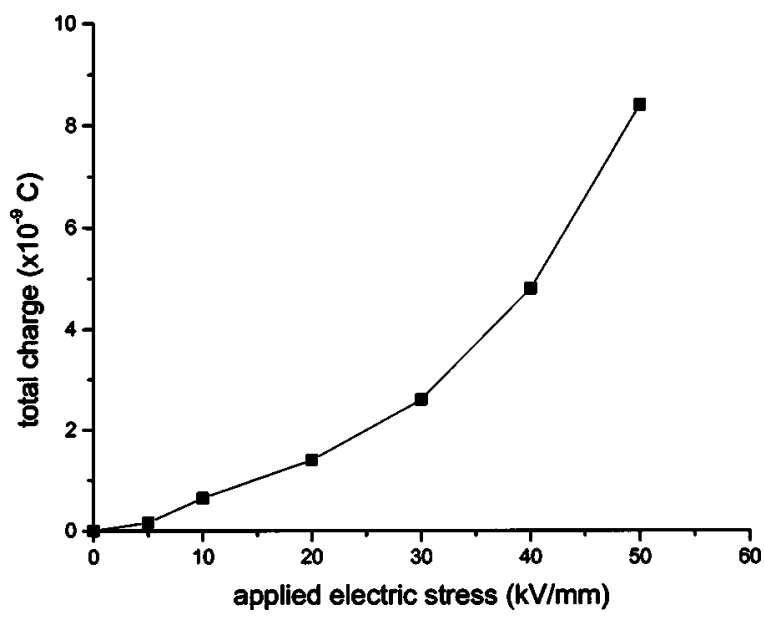

FIG. 5. Total charge in ac aged sample vs the applied ac stress.

sides were aged at $50 \mathrm{kV} / \mathrm{mm}$ for various times under the same conditions as those mentioned earlier. Space-charge distributions after the removal from the aging were measured and the results are shown in Fig. 6. Clearly, there was much less charge accumulated in the bulk of the sample in comparison with those aged with brass and semicon electrodes, indicating the significant influence of electrode material on charge formation in the bulk. It is also interesting to notice that the polarity of the charge is positive in nature.

\section{Space-charge characteristics under dc voltage}

In order to explore the possible effect brought in during the ac aging, space-charge dynamic characteristics under $\mathrm{dc}$ stress were also investigated. The sample was aged at 50 $\mathrm{kV} / \mathrm{mm}$ with brass and semicon electrodes for $40 \mathrm{~h}$ before it was removed from ac aging for dc stressing. The same dc stress level as ac aging stress, i.e., $50 \mathrm{kV} / \mathrm{mm}$ was applied to the aged sample. For comparison reason, charge dynamics in an unaged sample were also measured. Both charge accumulation during stressing and charge decay after the removal of dc stress were monitored. The electrodes used for applying dc were semicon as the anode and aluminum as the cathode.

The charge dynamics were complex and there was a significant change in charge distribution during the application

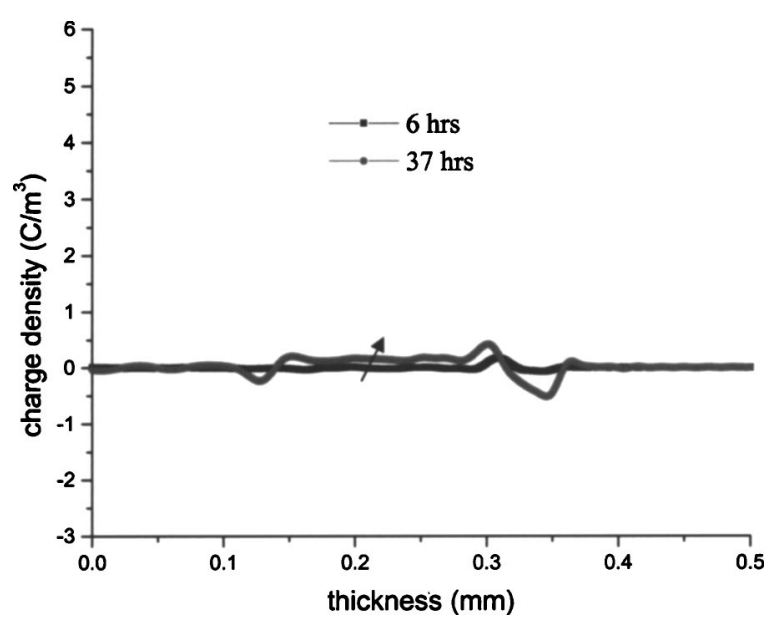

FIG. 6. Effect of Au electrode on charge accumulation in ac aged samples.

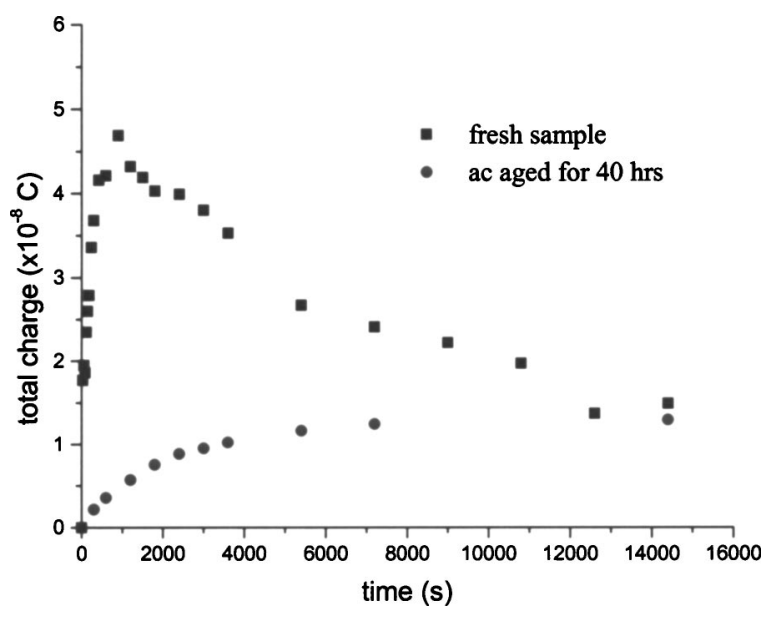

FIG. 7. Charge accumulation under $50-\mathrm{kV} / \mathrm{mm}$ dc electric stress.

of dc voltage. The detailed analysis of the results is in itself a separated issue and will not be presented here. In this paper only the total absolute amount of charge in the material is considered. The characteristics of the amount of charge formed in the bulk in the aged and unaged samples are very different as shown in Figs. 7 and 8 where the total charge accumulated in the samples under dc voltage is plotted as a function of dc stressing time for voltage on and decay time for voltage off, respectively.

From Fig. 7 it can be seen that the difference in the two samples is obvious during dc stressing period. The amount of charge in the ac aged sample increases with the duration of $\mathrm{dc}$ voltage application and then approaches to saturation. The amount of charge in the unaged sample increases rapidly at the beginning and reaches to its peak at $\sim 15 \mathrm{~min}$. After that, the amount of charge in the bulk starts to decrease and becomes stable in about $4 \mathrm{~h}$. Although the amount of charge at the early stage of dc stressing is significantly higher than the aged samples, the final amount after $4 \mathrm{~h}$ is comparable with the aged samples.

The total amount of charge as a function of time after the removal of the applied dc stress is shown in Fig. 8. Again there is a distinct difference in charge decay for aged and unaged samples. For aged sample the amount of charge re-

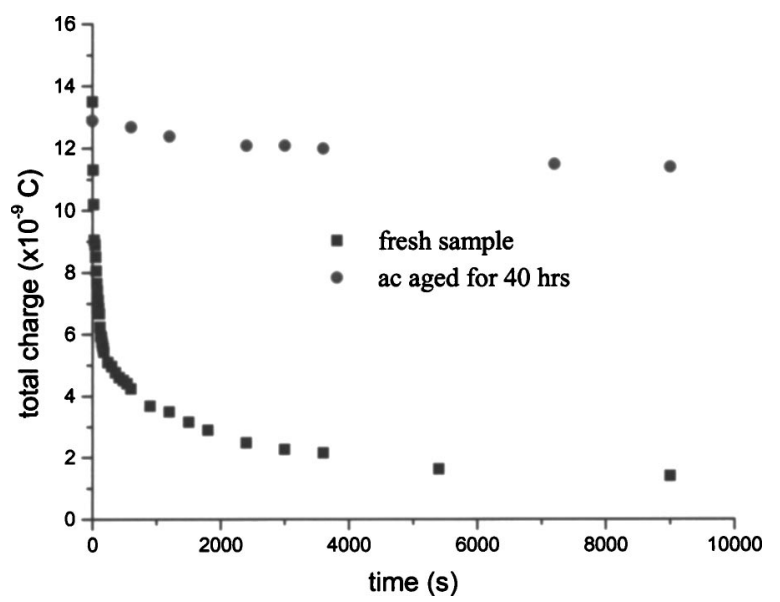

FIG. 8. Charge decay in ac aged and unaged samples after the removal from dc electric stress. 

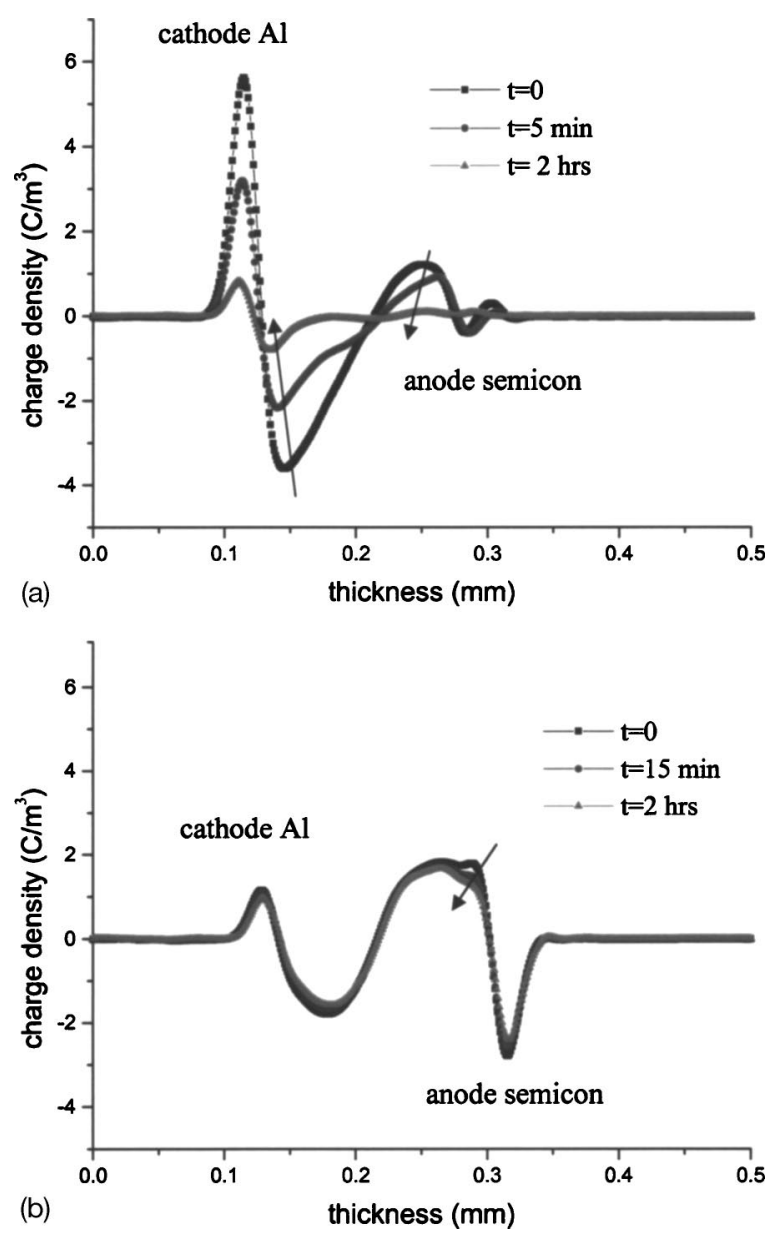

FIG. 9. Charge distribution at different times after the removal from 50$\mathrm{kV} / \mathrm{mm}$ dc electric stress for $4 \mathrm{~h}$ (a) unaged sample and (b) ac aged sample.

mains unchanged over a long period of time while for unaged sample there is a significant decrease in the first $15 \mathrm{~min}$ followed by a slow decay. The final amount of charge after 3-h decay indicates much less charge presence in the unaged sample.

The final charge distributions after the removal of dc stress for unaged and aged samples are illustrated in Fig. 9. In the case of ac aged sample, the charge distribution is more symmetric, i.e., similar amount of charge with negative charge adjacent to the cathode and positive charge to the anode. For unaged sample, more negative charge was formed adjacent to the cathode. It is evident that the charge distribution in aged sample changes little and this behavior is similar to that observed straightly after ac aging without any dc bias. In contrast, the charge in the unaged sample disappears rapidly after the removal of the applied voltage.

\section{Chemical analysis}

Under the influence of electric stress, chemical changes may take place. Both infrared (IR) and Raman spectra have been used to measure chemical changes in LDPE. There is no significant change in the IR spectra therefore it is not shown here. Figure 10 depicts the Raman spectra of LDPE prior to and after ac aging tests.

There are no significant changes that occurred in the sample aged for $45 \mathrm{~h}$. The possible explanation is that the

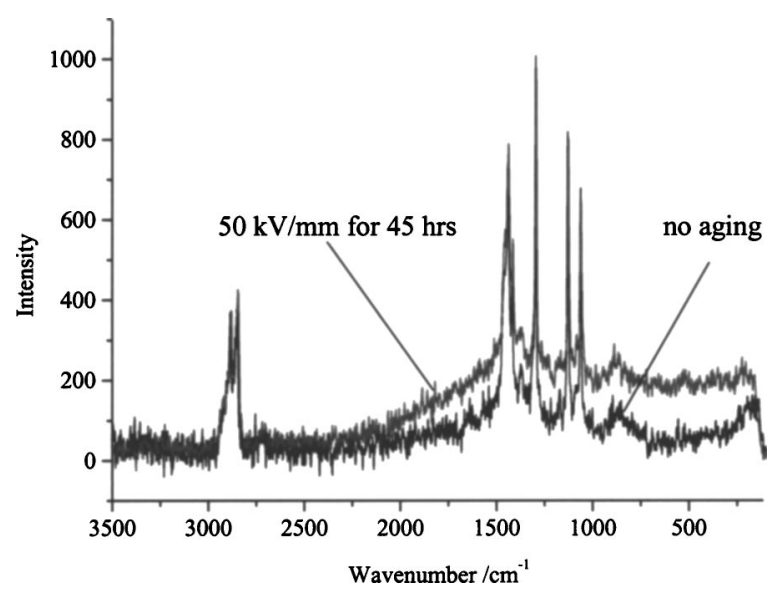

FIG. 10. Raman spectra of ac aged and unaged samples.

period of stressing time is too short. However, in the Raman spectra before and after aging, there is a slight rise in background which is caused by fluorescence. Recent investigation into electrical tree in polyethylene ${ }^{37}$ reveals that the fluorescence is due to partial degradation of the polymer. It must be stated that the rise in background is no way near to those observed in a treeing region.

\section{DISCUSSION}

The mechanisms of charge formation and its influence on the electrical performance of polymeric insulation are of technological importance. Despite the increasing body of evidence of space-charge presence under ac electric field in polymer insulation, the mechanism of charge generation and accumulation and its correlation with electrical aging under ac stress is still poorly understood. The results in Ref. 26 revealed that the frequency of the applied voltage plays a key role in the formation of space charge in polymeric insulation, while the study in Ref. 30 indicated that the space charge in the bulk of the sample aged under ac conditions at 40$\mathrm{kV} / \mathrm{mm}$ rms for 14 days is related to polymeric materials and the amount of charge depends on temperature. The electrodes used for study in Ref. 30 were graphites. Our results clearly demonstrate that the level of the applied voltage, the duration of the voltage application, and electrode materials are also important in determining the charge formation and its dynamics. Since additive-free LDPE was used in the present study, the impurities and their effect on space charge therefore should not be a major factor in the charge generation. Space charge accumulated in the sample can be directly attributed to the charge injection at the dielectric and electrode interface. This conclusion has also been proven by the absence of space charge when the sample was coated on both sides with gold electrodes. It has been revealed that gold electrode has low charge injection ability ${ }^{31}$ because of its high work function. When the same level of electric stress is applied to the sample, much less charge is observed in the case of gold electrodes, as shown in Fig. 6. It would show the similar charge distributions if the impurities were a dominant factor in charge formation. In fact, a small amount of positive charge was observed. This is consistent with our previous results. ${ }^{31}$ Different configurations of electrode ma- 
terials indicated that $\mathrm{Au}$ electrode predominately injects holes. Figure 5 clearly shows that the amount of injected charge increases with the applied ac aging stress. Assuming Schottky injection is the mechanism for the injection, the amount of charge injected into the bulk will increase rapidly with the electric field. The amount of charge accumulated is related not only to the injection rate but also to the trap density and its depth distribution. Although both types of charge can be injected into the bulk, the charge observed in the present arrangement shows only net total negative charge. This may be related to different mobilities possessed by two types of charges. The results in Ref. 31 seem to indicate that electrons have a higher mobility than the positive charges which means that the electrons can travel far deep into the bulk of the sample during the negative half cycle. On the other hand, the positive charge can only stay very close to the injecting electrode due to a slow mobility during the positive half cycle. Consequently the positive charges will be easily neutralized in the next negative half cycle. The stable charge distribution after ac aging, as shown in Fig. 4, indicates the presence of deep traps. Once the negative charges are trapped in these deep traps it is not easy for them to get detrapped. Therefore, over a period of time the net negative charge will be gradually accumulated in the bulk.

The existence of traps in semicrystalline polymeric materials is well recognized. ${ }^{38,39}$ They mainly locate at the interfaces between crystalline regions and amorphous regions. Extra traps can be formed by the fold at the surface in the long molecular chains. The depth of these traps varies depending on the nature of the traps. It has been reported to be in the range of $0.3-3 \mathrm{eV}$. As discussed earlier when the material is subjected to a high voltage, the charge injection may take place. The injected charge can be captured on the way towards the opposite electrode, so the traps in the material will be gradually filled up. Of course the exact amount of trapped charge in the bulk depends on the duration of the voltage application and the magnitude of the voltage as well as trap density and its depth distribution.

Based on the above description, the results shown in Figs. 7 and 8 indicate different trapping characteristics in ac aged and unaged samples. Due to rapid increase and decrease in the total absolute amount of charge it suggests that shallow traps govern the charge dynamics in the unaged sample. This is further supported by rapid decay after the removal of the external dc voltage. On the contrary, the trapping characteristics in the ac aged sample are dominated by the deep traps. The stable charge distribution implies that all the charges are trapped deeply. The evidence presented strongly suggests that the ac aging has changed the trapping characteristics of the material. One possible scenario is that ac aging converts some of the shallow traps into deep traps. The slight change in the Raman spectra indicates partial degradation of the material by ac aging. The detailed mechanisms of how aging causes the degradation are still an ongoing research but it is suspected that the injected charges may possess sufficient energy to break the chains of polyethylene, leading to formation of double bonds and other forms of chemical reactions. Although the change may not be signifi-

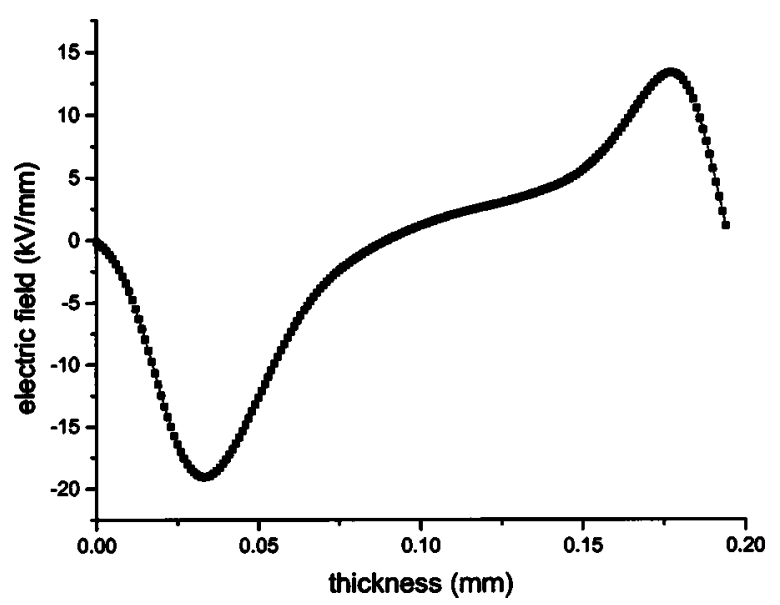

FIG. 11. Electric field produced by space charge accumulated during ac aging.

cant for detection by technique such as infrared spectroscope, it may be sufficient to alter the trapping characteristics of the material.

The above is partially supported by findings published by Mazzanti et al. ${ }^{40,41}$ in which they noticed that space charge in LDPE decreased slower and slower with dc aging time. This discovery also suggests that space-charge measurement is a very sensitive technique to monitor degradation.

Once the charge is trapped in the material it will then cause the distortion of the original field distribution. Charges formed in the material can be classified into fast and slow charges depending on their mobility. In most cases it is the slow charge (or deep-trapped charges) which may cause some adverse effect on the performance of the insulation, such as lifetime reduction of insulation due to local electric stress enhancement. The electric stress caused by space charge can be calculated using

$$
E(x)=\int_{0}^{x} \frac{\rho(x)}{\varepsilon_{0} \varepsilon_{r}} d x, \quad 0 \leqslant x \leqslant d .
$$

This is the case for ac aged samples where the charge formed after ac aging is extremely stable. Based on the charge distribution shown in Fig. 2, the electric stress distribution due to charge is illustrated in Fig. 11. The boundary condition $V=\int_{0}^{d} E(x) d x=0$ was imposed as the space-charge measurements were performed under short-circuit condition. The maximum stress for the sample aged for $2 \mathrm{l} \mathrm{h}$ occurs at the interfaces. The estimated field is $\sim 20 \mathrm{kV} / \mathrm{mm}$, comparable with the aging stress $(50 \mathrm{kV} / \mathrm{mm})$. This has a significant implication for insulation systems. Because of charge stability the electric stress at the interface in one of the half cycles can be as high as $70 \mathrm{kV} / \mathrm{mm}$. It is known that the high local electric stress can lead to partial discharge activity, resulting in degradation of the material and possibly premature failure of the system.

An estimate can be made of the consequence of such stress enhancement (due to the presence of space charge) on the material's life using the inverse power law 


$$
E^{n} t=\text { const, }
$$

where $E$ is the electric stress experienced by the material, $t$ the expected lifetime of the material, and. $n$ is a constant. For example, in view of the above estimation, the electric stress increased by $40 \%$, hence the lifetime is reduced to $6.8 \%$ of the original $(1 \mathrm{pu})$ with a conservative value $n=8$. This is a significant reduction in lifetime for the insulation.

\section{CONCLUSIONS}

The work presented in this paper concerns with the space-charge accumulation in LDPE samples after electrically aged at $50 \mathrm{~Hz}$. It has been experimentally demonstrated that the space charge under ac aging conditions is related to the level of the applied field, duration of the voltage application, as well as the electrode materials. The results unambiguously indicate the presence of space charge under ac conditions. Several findings are significant. By comparing with the results of the unaged sample the results from the aged sample provide a direct evidence of changing trapping characteristics after ac aging. The effect of electrode materials suggests that by selecting a proper electrode material, the amount of charge injected into the bulk can be significantly reduced and this will lead to less degradation of the material. More specifically, the following conclusions may be drawn from the study.

(1) Negative space charge is present in the bulk of the material and the total amount of charge increases with the aging time. The amount of charge increases with the applied field. Decay test indicates that the charges are captured in deep traps. These deep traps are believed to form during the aging and related to change caused by injected charge. Electrode material has an important role in the formation of charge, hence subsequent changes caused by charge.

(2) Chemical analysis shows no detectable changes taken place in the bulk. This may suggest that the space-charge measurement is a very sensitive technique which can reveal minor physical and chemical changes in the aged material.

(3) The charge dynamics of ac aged LDPE under dc stress are significantly different from that observed in unaged material. Much stable charge formation in ac aged sample supports the formation of deep traps.

(4) The presence of deeply trapped space charge leads to an electric stress enhancement which may shorten the lifetime of the insulation system.

${ }^{1}$ G. Chen and A. E. Davies, IEEE Trans. Dielectr. Electr. Insul. 7, 401 (2000).

${ }^{2}$ H. Wagner, Ann. Rep. Conf. Elec. Insul. Dielectr. Phenom. 62 (1975).

${ }^{3}$ S. N. Kolesov, IEEE Trans. Electr. Insul. 15, 382 (1980).

${ }^{4}$ L. Y. Gao, D. M. Tu, S. C. Zhou, and Z. L. Zhang, IEEE Trans. Electr Insul. 25, 535 (1990).
${ }^{5}$ A. Gustafsson, M. T. Conde Brana, and U. W. Gedde, Polymer 32, 426 (1991).

${ }^{6}$ L. A. Dissado, I. Doble, S. V. Wolfe, A. E. Davies, and G. Chen, IEEE Trans. Dielectr. Electr. Insul. 4, 1 (1997).

${ }^{7}$ T. Tanaka, T. Okamoto, N. Hozumi, and K. Suzuki, IEEE Trans. Dielectr. Electr. Insul. 3, 345 (1996).

${ }^{8}$ J. Artbauer, J. Phys. D 29, 446 (1996).

${ }^{9}$ M. A. Brown, G. Chen, A. S. Vaughan, and P. A. Norman, J. Phys. D 36, 3197 (2003).

${ }^{10}$ R. Bartnilas, IEEE Trans. Dielectr. Electr. Insul. 4, 544 (1997).

${ }^{11}$ G. Blaise, J. Appl. Phys. 77, 2916 (1995).

${ }^{12}$ N. Shimuzu, Ph.D. thesis, Faculty of Engineering, Nagoya University, Japan, 1979.

${ }^{13}$ M. A. Brown, G. Chen, and P. A. Norman, J. Phys. D 36, 3191 (2003).

${ }^{14} \mathrm{G}$. Chen, Proceedings of the Second ICMEP, Changging, China, 2003 (Sichun Publishing House of Science and Technology, Chongqing, 2003), p. 133.

${ }^{15}$ M. A. Brown, G. Chen, A. E. Davies, L. A. Dissado, and P. A. Norman, IEEE Trans. Dielectr. Electr. Insul. 7, 346 (2000).

${ }^{16}$ B. Sanden, Ph.D. thesis, Norwegian University of Science and Technology, 1996.

${ }^{17}$ T. Mizutani, IEEE Trans. Dielectr. Electr. Insul. 1, 923 (1994)

${ }^{18}$ X. Wu, G. Chen, A. E. Davies, R. N. Hampton, S. J. Sutton, and S. G. Swingler, IEEE Trans. Dielectr. Electr. Insul. 8, 725 (2001).

${ }^{19}$ X. Wang, D. Tu, Y. Tanaka, T. Muronaka, T. Takada, C. Shinoda, and T. Hashizumi, IEEE Trans. Dielectr. Electr. Insul. 2, 467 (1995).

${ }^{20}$ K. R. Bambery and R. J. Fleming, J. Therm. Anal. 50, 19 (1997).

${ }^{21}$ L. A. Dissado, G. Mazzanti, and G. C. Montanari, IEEE Trans. Dielectr. Electr. Insul. 4, 496 (1997).

${ }^{22}$ P. Notingher, Jr., A. Toureille, J. Santana, and M. Albertini,Jicable, Versailles, France, 20-24 June 1999, p. 701.

${ }^{23}$ S. Agnel, A. Toureille, G. Platbrood, and G. Geert, Jicable, Versailles, France, 20-24 June 1999, p. 656.

${ }^{24}$ S. Hole, C. Alquié, and J. Lewiner, C. R. Acad. Sci., Paris 316, Série II 157 (1993).

${ }^{25}$ M. Mammeri, C. Laurent, and J. Salon, IEEE Trans. Dielectr. Electr. Insul. 2, 27 (1995).

${ }^{26}$ T. Takada, IEEE Trans. Dielectr. Electr. Insul. 6, 519 (1999).

${ }^{27}$ Y. F. F. Ho, G. Chen, A. E. Davies, S. G. Swingler, S. J. Sutton, R. N. Hampton, and S. Hobdell, IEEE Trans. Dielectr. Electr. Insul. 9, 362 (2002).

${ }^{28}$ C. Bert, C. Heninion, J. Lewiner, C. Alquié, N. Hampton, J. Freestone, and S. Verne, Jicable, Versailles, France, 1995, p. 195.

${ }^{29}$ O. Fanjeau, D. Mary, and D. Malec, J. Phys. D 33, L61 (2000).

${ }^{30}$ P. Notingher, Jr., A. Toureille, J. Santana, L. Martinotto, and L. Albertini, IEEE Trans. Dielectr. Electr. Insul. 8, 972 (2001).

${ }^{31}$ G. Chen, T. Y. G. Tay, A. E. Davies, Y. Tanaka, and T. Takada, IEEE Trans. Dielectr. Electr. Insul. 8, 867 (2001).

${ }^{32}$ G. Chen, Y. Tanaka, T. Takada, and L. Zhong, IEEE Trans. Dielectr. Electr. Insul. 11, 113 (2004).

${ }^{33}$ Y. Li, M. Yasuda, and T. Takada, IEEE Trans. Dielectr. Electr. Insul. 1, 188 (1994).

${ }^{34}$ M. Fu, G. Chen, and A. E. Davies, IEE Proc.: Sci., Meas. Technol. 150, 89 (2003).

${ }^{35}$ T. Maneo, T. Futami, H. Kushibe, T. Takada, and C. M. Cooke, IEEE Trans. Electr. Insul. 23, 433 (1988).

${ }^{36}$ A. Vazquez, G. Chen, A. E. Davies, and R. Bosch, J. Eur. Ceram. Soc. 19, 1219 (1999).

${ }^{37}$ A. S. Vaughan, S. J. Dodd, and S. J. Simon, J. Mater. Sci. 39, 181 (2004).

${ }^{38}$ L. A. Dissado and J. C. Fothergill, Electrical Degradation and Breakdown in Polymers (Peter Peregrinus Ltd., London, 1992).

${ }^{39}$ M. Meunier, N. Quirke, and A. Aslanides, J. Chem. Phys. 115, 2876 (2001).

${ }^{40}$ G. Mazzanti, G. C. Montanari, and F. Palmieri, IEEE Trans. Dielectr. Electr. Insul. 10, 108 (2003).

${ }^{41}$ G. Mazzanti, G. C. Montanari, and J. M. Alison, IEEE Trans. Dielectr. Electr. Insul. 10, 187 (2003). 